\title{
Synergic Development of Energy Efficacious Wireless Sensor Networks
}

Shahzad Ashraf ( $\square$ nfc.iet@hotmail.com )

hohai university changzhou https://orcid.org/0000-0002-7637-7870

\section{Research Article}

Keywords: Wireless sensor network, distributed computing, synergic development, delay factor

Posted Date: April 22nd, 2020

DOl: https://doi.org/10.21203/rs.3.rs-23955/v1

License: (c) (i) This work is licensed under a Creative Commons Attribution 4.0 International License. Read Full License 


\title{
Synergic Development of Energy Efficacious Wireless Sensor Networks
}

\author{
Shahzad Ashraf \\ College of IoT, Hohai University Changzhou
}

\begin{abstract}
Considering Wireless Sensor Networks (WSNs) in today's scenario, sending and receiving uninterrupted sensory data remains a challenge to achieve with minimal latency and energy consumption as low as possible. Energy consumption is exponentially growing in computing devices such as computers, embedded systems, portable devices, and wireless sensor networks. Extensive research has been in practice recently to minimize energy consumption without compromising the Quality of Service (QoS) that is to provide data to the requester node with minimum Delay and high Reliability. This article analyzes the Synergic development of energy efficacious wireless sensor networks (SDEE) which aims to reduce energy demand by reducing the overhead packet in the network and also provides the requester with a minimum delay by collecting data requests from the next cache node in the vicinity of the requester or sink node. The findings of the simulation clearly show that energy usage is lower when the empirical grid model is used against the star / cluster model when the parameters are the same.
\end{abstract}

Keywords: Wireless sensor network, distributed computing, synergic development, delay factor

\section{Introduction}

A network technology is developed by the ZigBee Partnership, a multi-supplier group. The network layer ZigBee supports the topologies of stars, tree and mesh. In realistic implementations such as domestic control, smart transport systems, personal health care and military communications, ZigBee powered mesh networks are best adapted to. ZigBee maintains a low traffic rate but has intensified energy consumption leading to traffic packet transceiving, idling listening, overhearing, crashes and multi-cast overhead packets [1]. To order to achieve effective resources (for example, bandwidth and energy consumption), an optimal routing scheme is thus essential in a mesh network. Ashraf et al in [1] had previously explored the probability of achieving energy performance in these AODV routing protocol networks.

The proposed (SDEE) protocol with energy usage in ZigBee presents an improved solution for achieving better routing and energy efficiency by incorporating the localized caching algorithm that is the Caching in Cooperative Zones (CCZ) to overcome the limitation of AODV for energy efficiency usage. The rest of this paper is organized as follows. In Section II, the previous works related to the proposed scheme is discussed. Later in section III the proposed SDEE routing protocol in ZigBee is presented, and then our simulation model and parameters are discussed in Section IV. In Section V, the various simulation results are presented and also we compare the simulation results of the SDEE_CCZ with the existing AODV_CCZ. Conclusions are drawn in the last section.

\section{Literature Review}

Many garner systems have been suggested in the past before P.Charan et al.[1] introduced a caching system to be included in ZigBee-based AODV routing protocol networks. They had evaluated the performance of the proposed algorithm in two analytical models that are star based network model 
and the grid based network model respectively. Previously, Jinbao Li et al. [2] proposed a caching scheme in a sensor network of multisink environment. In their approach, the network forms a set of network trees for a particular receiver. A common subtree is formed from these tree sets, and the root of the common subtree is selected as a data cache node to reduce communication costs.

J. Xu et al. [3] proposes a wait-caching scheme that expects data from the same cluster to become available within a threshold, then aggregates it with packets from the lower cluster and later sends it to the receiver, thereby reducing the number of packets transmitted. Md.A. Rahman et al. [4] proposed an efficient caching mechanism which negotiates data between the base station and the sensor, resulting in an expectation of data change and data loss.

T.P. Sharma et al. [5] proposed a cooperative caching scheme that used cooperation between various SNs in the defined area. In addition to its own local storage, nodes also use node storage from other clusters around them to form larger cache storage, called cumulative Cache. In this case, a token-based cache admission control (CAC) scheme is designed in which the node holding the token can cache or replace the data item. One of the disadvantages of the proposed model is that it generates a larger amount of packet overhead to maintain and rotate the token.

\section{Synergic development of Proposed (SDEE) protocol}

The SDEE protocol is an energy aware routing protocol that is an extension to the popular AODV routing protocol. The route discovery process in SDEE is different from AODV. The SDEE protocol is capable of generating routing paths based on the residual energy of nodes in the sensor network. SDEE calculates the routing path based on the Threshold value that is set in the protocol as $80 \%$ of the Average Energy of the Network. The Average energy of the network depends on the Total Link Cost that appears between two nodes ' $i$ ' and ' $j$ ' while transmitting data from the source node ' $i$ ' to the destination node ' $j$ '.

Fig.1. ZigBee based network in which data is transmitted from source node ' $i$ ' to destination or sink node ' $j$ '

The estimation of the Total Link Cost can be achieved in the following manner wherein, a sensor network based on the IEEE 802.15.4 standard is considered in which the traffic associated with the $i$ th mote be ' $\boldsymbol{T}_{\boldsymbol{i}}$ ' packets per second (pps). Let us assume that a data flow ' $l$ ' carries data traffic at a rate of ' $\boldsymbol{t}_{\boldsymbol{l}}$ 'pps, then $\boldsymbol{T}_{\boldsymbol{i}}$ can be estimated as:

$$
\boldsymbol{T}_{\boldsymbol{i}}=\sum_{l \in D(i)} t_{l}
$$

Where, $D_{i} \rightarrow$ denotes the set of data flows through node ' $i$ '.

The two important metrics for performance evaluation of a sensor network based on IEEE802.15.4 are i). Power Consumption, and ii). Quality of Services (QoS). Let $\boldsymbol{P}_{\boldsymbol{i}}(T)$ and $\boldsymbol{Q}_{\boldsymbol{i}}(T)$ be the power requirement and the Quality of Service requirements of a particular node ' $i$ ' in the network when it carries $\mathrm{T}(\mathrm{pps})$.

If a new stream of data ' $m$ ' is added to node ' $i$ ' then this will result in a change in power consumption and QoS at that node.

Assuming that $\boldsymbol{p}_{\boldsymbol{i}}(T)$ be the instantaneous power consumption by the i-th node when it carries ' $\mathrm{T}$ ' packets per second that includes all aspects of packet processing: storing, routing and forwarding them through the neighboring nodes.

We now define the power cost asscoiated with the $m$-th data stream as $\boldsymbol{p}_{i}^{m}\left(t_{m}, T_{i}\right)$ at node ' $i$ ' which represents the power consumption of the m-th flow in combination with the power associated with other flows.So,

$$
\boldsymbol{p}_{i}^{m}\left(t_{m}, T_{i}\right)=a p_{i}\left(T_{i}+t_{m}\right)+b\left[p_{i}\left(T_{i}+t_{m}\right)-p_{i}\left(T_{i}\right)\right]
$$


Where; $a, b \geq 0$. The first term is total power in ( $\mathrm{mW}$ ) due to adding the $\mathrm{m}$-th flow, multiplied by some constant $a$. Whereas, the second term represents the increase in energy for the other flows, multiplied by some constant $b$.

Hence the total power cost functions for the $m$-th data flow of rate ' $t_{m}$ ' on a routing path ' $\lambda(i)$ 'starting from node ' $i$ ' is written as:

$$
P_{\lambda(i)}^{m}\left(t_{m}, \overline{T_{\lambda(i)}}\right)=\sum_{n \in \lambda(i)} P_{n}^{m}\left(t_{m}, T_{n}\right)
$$

Similarly, the Quality of Service criterion, such as packet loss, delay or Packet Delivery Ratio (PDR) is given by-

$$
Q_{\lambda(i)}^{m}\left(t_{m}, \overline{T_{\lambda(i)}}\right)=\sum_{n \in \lambda(i)} Q_{n}^{m}\left(t_{m}, T_{n}\right)
$$

Where $\overline{T_{\lambda(i)}}=\left(T_{n_{1}}, T_{n_{2}}, \ldots \ldots, T_{n_{|\lambda(i)|}}\right)$ where $n_{l}=i$ and $n_{j}$ are successive nodes in the routing path within the range $1 \leq j \leq|\lambda(i)|$.

Since, SDEE protocol is expected to minimize the overall cost of power consumption while satisfying the requested QoS, therefore, it is essential to optimize the total energy cost attribute, $\mathrm{TEC}_{i}$ which will be a resultant of the power and the Quality of Service constraint. Hence, the total energy cost, $\mathrm{TEC}_{i}$ will be represented as:

$$
\operatorname{TEC}_{i}=P_{\lambda(i)}^{m}\left(t_{m}, \overline{T_{\lambda(i)}}\right)+\gamma Q_{\lambda(i)}^{m}\left(t_{m}, \overline{T_{\lambda(i)}}\right)
$$

Where, $\gamma>0$ is a constant that matches the delay units with respect to power.

A sensor node will only be added to the routing path in a Distributed Energy Aware Routing (SDEE) protocol if the Threshold attribute is considered as a checkpoint. The node with $80 \%$ or more residual energy of the total energy cost will be included in a probable routing path. Thus for a data to be sent from source node ' $i$ ' to the destination node ' $j$ ' the threshold energy of the battery of nodes falling in its routing path is represented by-

$$
\text { Threshold }=0.8 \times T E C_{i j}
$$

\section{Simulation Model and Parameters}

The proposed CCZ algorithm is simulated on NS-2(version 2.32). The routing protocols used is SDEE for routing data traffic in a WSN based on the IEEE 802.15.4 PHY and MAC protocols and a free-space propagation model as a radio propagation model. The number of nodes in the network is 16 , and it is deployed in a $100 * 100$ square meter sensor area. The wireless bandwidth is $250 \mathrm{kbps}$, which represents the maximum amount of data or bits that can be transmitted over a period of time, typically one second.

In Star (Cluster based) network consists of 16 sensor nodes based on IEEE 802.15.4 PHY and MAC standard are considered which are all Full Function devices (FFDs) and are accompanied with a fixed amount of cache memory to store the sensed data as shown in figure $3 \mathrm{a}$. 


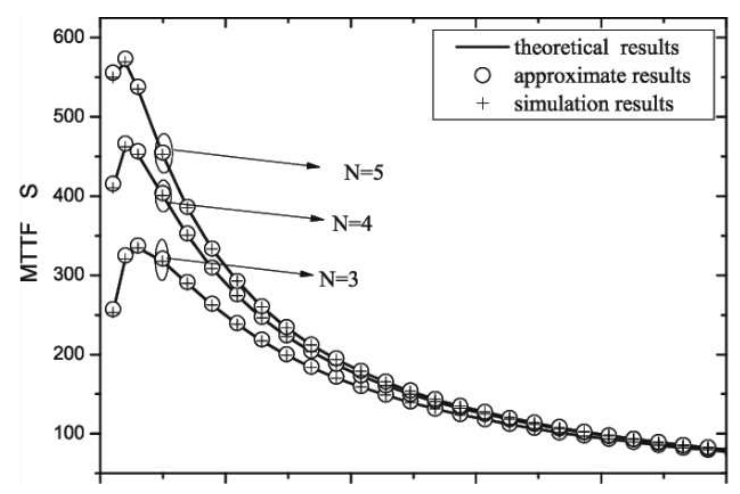

Fig. 3a. Star Based Network Model

In Grid (peer-to-peer) based network, sixteen sensor nodes are placed equidistant in an area of $100 \mathrm{~m} * 100 \mathrm{~m}$, and all devices are IEEE 802.15.4 PHY and MAC compliant, as shown in figure $3 \mathrm{~b}$. All devices are associated with some fixed amount of cache to store and update it with fresh data.

The IEEE 802.15.4 based sensor motes can generally be in one of the following modes: Idle, Transmit Mode, or Receive Mode. In the simulation we set the $\mathrm{BO}=3$ and the $\mathrm{SO}=2$, for which the Duty Cycle comes out to be:

Duty cycle $=2^{-(\mathrm{BO}-\mathrm{SO})}=2^{-(3-2)}=2^{-1}=\frac{1}{2}=0.5$ or $50 \%$. A sensor node with very low duty cycle goes to sleep mode until a beacon signal arrives again to wake up the radio[6].

The data items are updated at the source nodes. The source node serves the requests on First-ComeFirst-Serve (FCFS) Basis.

The following three performance metrics have been evaluated:

\section{A. Average Query Latency $\left(T_{q_{\text {avg }}}\right)$}

The query latency $\mathbf{T}_{\boldsymbol{q}}$ ' can be defined as the time interval between a query sent by a requester and the response received back by the requester/sink. The average query latency is the query latency ' $\boldsymbol{T}_{\boldsymbol{q}}$ ' averaged over all the generated queries.

\section{B. Byte Hit Ratio(B)}

The byte hit ratio $\boldsymbol{B}$ is defined as the ratio of total bytes of data retrieved from the cache to the total number of requested data bytes by the sink/requester node. The byte hit ratio $\boldsymbol{B}$ includes bytes retrieved from a local hit known as a Local Byte $\operatorname{Hit}\left(\boldsymbol{B}_{\text {local }}\right)$; bytes retrieved from a zone hit known as a Zone Byte Hit $\left(\boldsymbol{B}_{\text {zone }}\right)$ and the bytes retrieved from a remote hit known as a Remote Byte $\operatorname{Hit}\left(\boldsymbol{B}_{\text {remote }}\right)$.It is to be noted that bytes retrieved from a Global hit is a freshly sensed data and cannot be considered to have been retrieved from the cache.

\section{Total Energy Consumption $\left(E_{\text {total }}\right)$}

Total energy consumption is defined as the algebraic sum of energy consumed by each IEEE 802.15.4/ ZigBee sensor mote during transmission, reception, and sleep modes. For simulation purpose, we consider the unit of energy consumption in $\mathrm{mWh}$.

$$
E_{\text {total }}=E_{\text {transmit }}+E_{\text {receive }}+E_{\text {sleep }}
$$




\section{Simulation Results}

Figure $4 \mathrm{a}$ shows the effect of varying cache sizes on the Average Query Latency for both the analytical network models that is Star/ Cluster connected Network and the Grid Based Network. Simulation shows that the average query latency $\left(T_{q_{a v g}}\right)$ decreases as the size of the cache increases, since both cases satisfy more number of data queries. This is because as the size of the cache increases, more of the required data items can be found in the local cache. Average query latency in grid/peer-to-peer networking is less than in Star / Cluster based networks. Therefore, all of the CCZ caching algorithms in Grid Connected Network perform better than Star Connected Network.

the impact of varying cache size on byte hit ratio. Both the Star Connection Network and the Grid Connection Network show better byte hit rates as the size of the cache increases. When the cache size is small, the data is more contributed by zone hits and remote hits, but as the cache size is increased, the contribution of local hits becomes significant. This is because as the cache gets larger, a larger number of data items are found in the local cache. The local byte hit rate increases as the size of the cache increases because more data can be cached locally for larger cache storage. According to the simulation results, the grid connection network has better byte hit ratio than the star / cluster connection network.

Figure 5a shows the total energy consumption of nodes in a network connected by a star (cluster) connection. Different data rates are considered in the range of 1 to 5 packets per second (pps). Two scenarios are considered in the analysis of energy consumption, in one case the SDEE routing protocol is considered and in the other case the SDEE routing protocol is used in cooperation with the $\mathrm{CCZ}$ cache algorithm in order to minimize the packet transmission delay and therefore the overall power consumption. Based on the results, we see that when the data rate is 5 pps, the node consumes less energy when combined with the caching algorithm; this is because the requester retrieves data from a nearby cache node or the local hit may have occurred, and the data item is retrieved from the requesting node itself. For the Star/Cluster network with SDEE routing protocol, the average node energy consumption is $2.27 \mathrm{mWh}$. However, when SDEE routing protocol and CCZ cache algorithm are used together, the average energy consumption is $1.77 \mathrm{mWh}$.

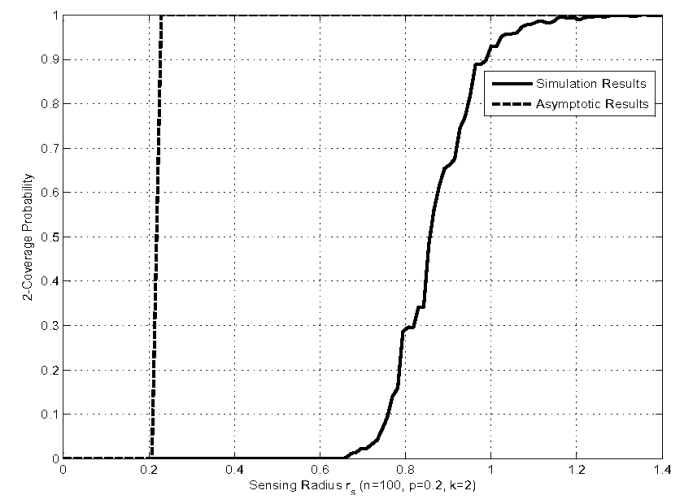

Fig. 5.a. Comparison of Energy Consumption in Star/Cluster Network for SDEE routing with and without $\mathrm{CCZ}$ caching algorithm

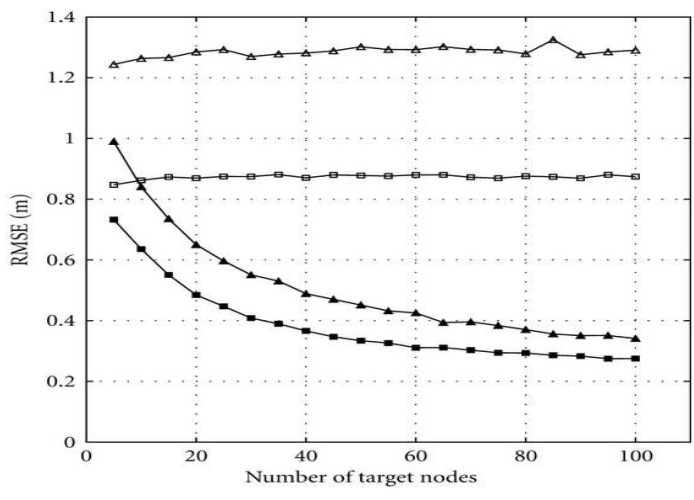

Fig. 5.b. Comparison of Energy Consumption in Grid/peer-to-peer Network for SDEE routing with and without $\mathrm{CCZ}$ caching algorithm. 
Figure $5 \mathrm{~b}$ shows the total energy consumption of nodes when connected in a grid (peer-to-peer) connection. Different data rates are considered in the range of 1 to 5 packets per second (pps). The similar scenarios are considered while analyzing for energy consumption, that is one in which SDEE routing protocol is considered and in the other scenario, SDEE routing protocol is simulated with the CCZ Cooperative Caching Algorithm. The simulation results show that when only SDEE routing protocol is alone working then the energy consumption is increasing as the data rate is increased. However, when the $\mathrm{CCZ}$ caching algorithm comes into action with the SDEE routing protocol then the Energy Consumption continues to drop. For a Grid Network, the average energy consumption by nodes with only SDEE routing algorithm was $2.24 \mathrm{mWh}$. And when, SDEE routing protocol is used with the CCZ caching algorithm then the average energy consumption is $1.12 \mathrm{mWh}$.

\section{Comparison of SDEE_CCZ with AODV_CCZ}

\section{A. For Star/Cluster based Network}

When AODV routing protocol is simulated with the localized $\mathrm{CCZ}$ caching algorithm then the average energy consumption was $2.43 \mathrm{mWh}$ for cluster network [1], whereas for the same network when SDEE routing protocol with CCZ algorithm is simulated then the energy consumption is $1.77 \mathrm{mWh}$. This result proves about the efficacy of the localized algorithm wherein the requester gets the requested datum from the nearby cache via the local hit, zone hit, remote hit or the global hit.

\section{B. For Grid/Peer-to-Peer based Network}

When AODV routing protocol is simulated with the localized CCZ caching algorithm then the average energy consumption was $1.92 \mathrm{mWh}$ for grid network [1], whereas for the same network when SDEE routing protocol with CCZ algorithm is simulated then the energy consumption is $1.12 \mathrm{mWh}$. This shows that the algorithm is serving the data to the requester by providing the data stored in the cache memory locally or remotely in a particular zone, thereby providing data to the source with minimal latency and higher accuracy.

\section{Conclusion}

The CCZ algorithm ensures that a query is served from the nearest cache or source. Simulation results show that the CCZ caching scheme performs better in grid based or peer-to-peer network model than the cluster based or star network model for SDEE routing protocol.

\section{References:}

[1] Q. Zhu, J. Sarkis, "Relationships between operational practices and performance among early adopters of green supply chain management practices in Chinese manufacturing enterprises", Journal of Operations Management, Vol. 22, No. 3, pp. 265-289, 2004

[2] Ashraf, S., Gao, M., Mingchen, Z., Ahmed, T., Raza, A., \& Naeem, H. (2020). USPF: Underwater Shrewd Packet Flooding Mechanism through Surrogate Holding Time. Wireless Communications and Mobile Computing, 2020, 112.doi:10.1155/2020/9625974

[3] C. I. Yang, S. Lien, "Governance mechanisms for green supply chain partnership", Sustainability, Vol. 10, No. 8, Article ID 2681, 2018

[4] A. Longoni, D. Luzzini, M. Guerci, "Deploying environmental management across functions: The relationship between green human resource management and green supply chain management", Journal of Business Ethics, Vol. 151, No. 4, pp. 1081-1095, 2018

[5] Ashraf, S; Ahmed, Tauqeer; Raza, Asif; Naeem, Hamad. 2020. "Design of Shrewd Underwater Routing Synergy Using Porous Energy Shells." Smart Cities 3, no. 1: 74-92. doi: 10.3390/smartcities3010005

[6] C. Busse, J. Meinlschmidt, K. Foerstl, "Managing information processing needs in global supply chains: A prerequisite to sustainable supply chain management”, Journal of Supply Chain Management, Vol. 53, No. 1, pp. 87-113, 2017 
[7] Ashraf, S; Mingsheng Gao, Zhengming Chen, Syed Kamran Haider and Zeeshan Raza, "Efficient Node Monitoring Mechanism in WSN using Contikimac Protocol" International Journal of Advanced Computer Science and Applications(IJACSA), 8(11), 2017. doi:10.14569/IJACSA.2017.081152

[8] Lean and Green Supply Chain Management. (2019). In T. Paksoy, G.-W. Weber, \& S. Huber (Eds.), International Series in Operations Research \& Management Science. https://doi.org/10.1007/978-3-319-97511-5

[9] M. S. Shahbaz, R. Z. R. M. Rasi, M. H. Zulfakar, M. F. B. Ahmad, M. Asad, "Theoretical framework development for supply chain risk management for Malaysian manufacturing”, International Journal of Supply Chain Management, Vol. 7, No. 6, pp. 325-338, 2018 
Figures

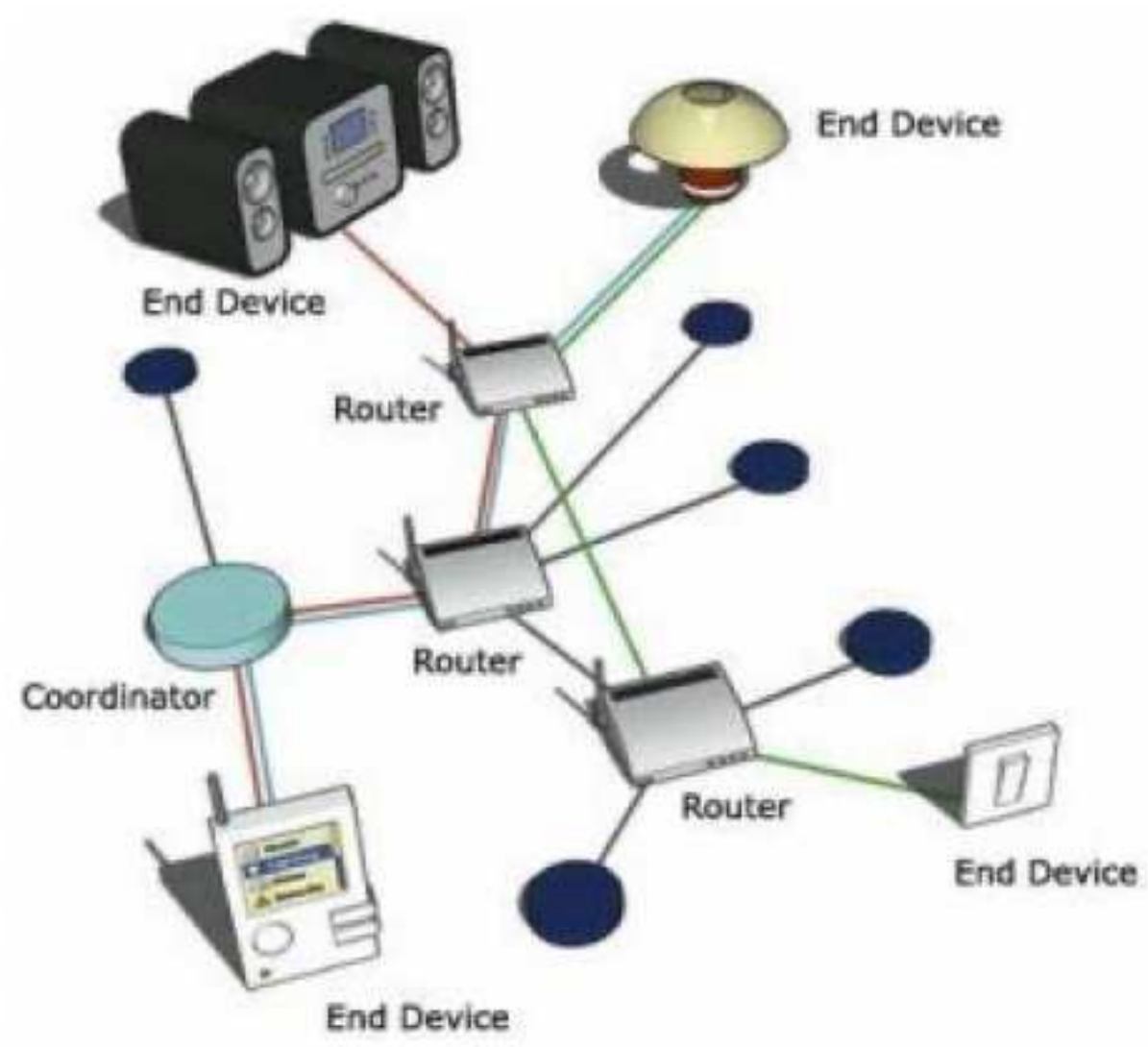

Figure 1

Network wth ZigBee operation

Figure 2 was omitted by the authors in this version of the paper.

Figure 2 
A

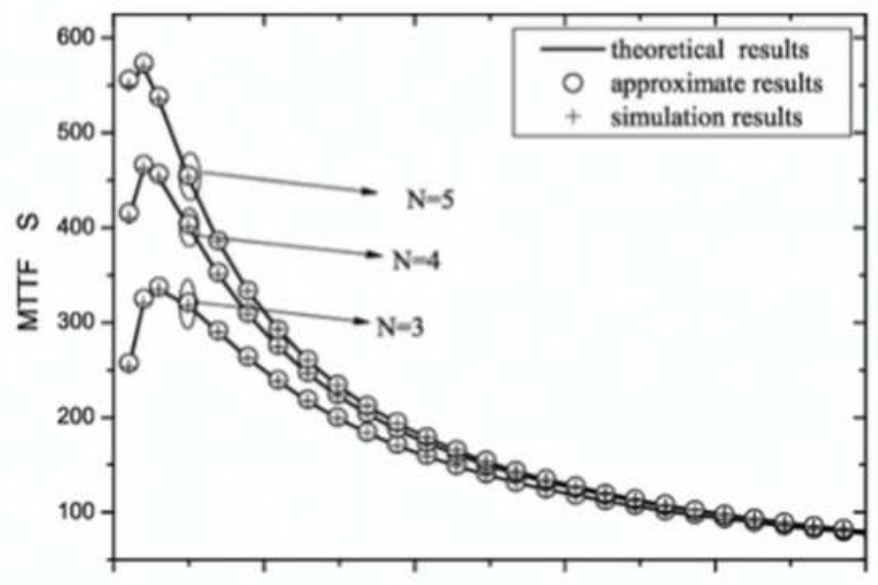

B

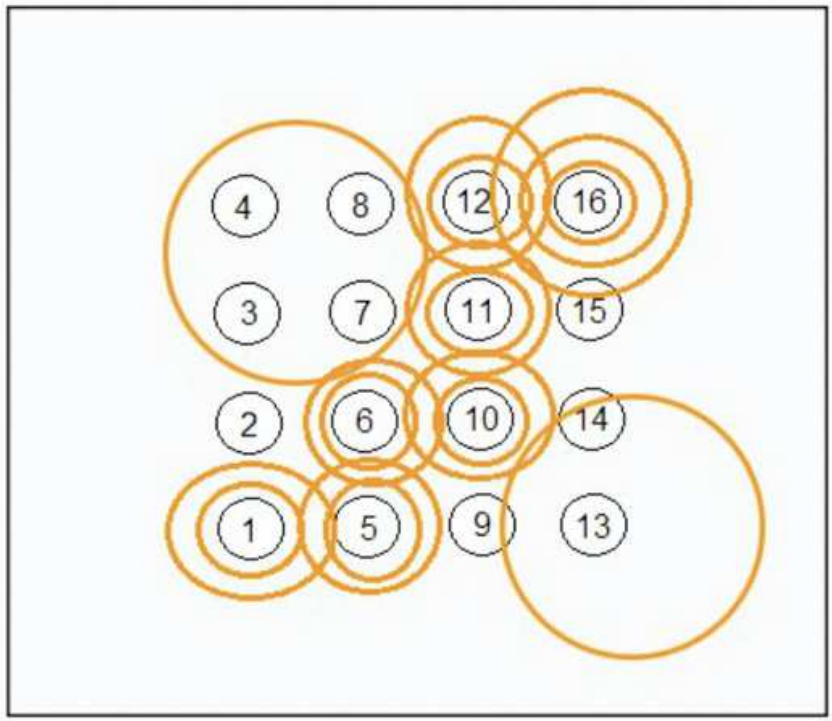

Figure 3

A. Star Based Network Model. B. Network topological operation

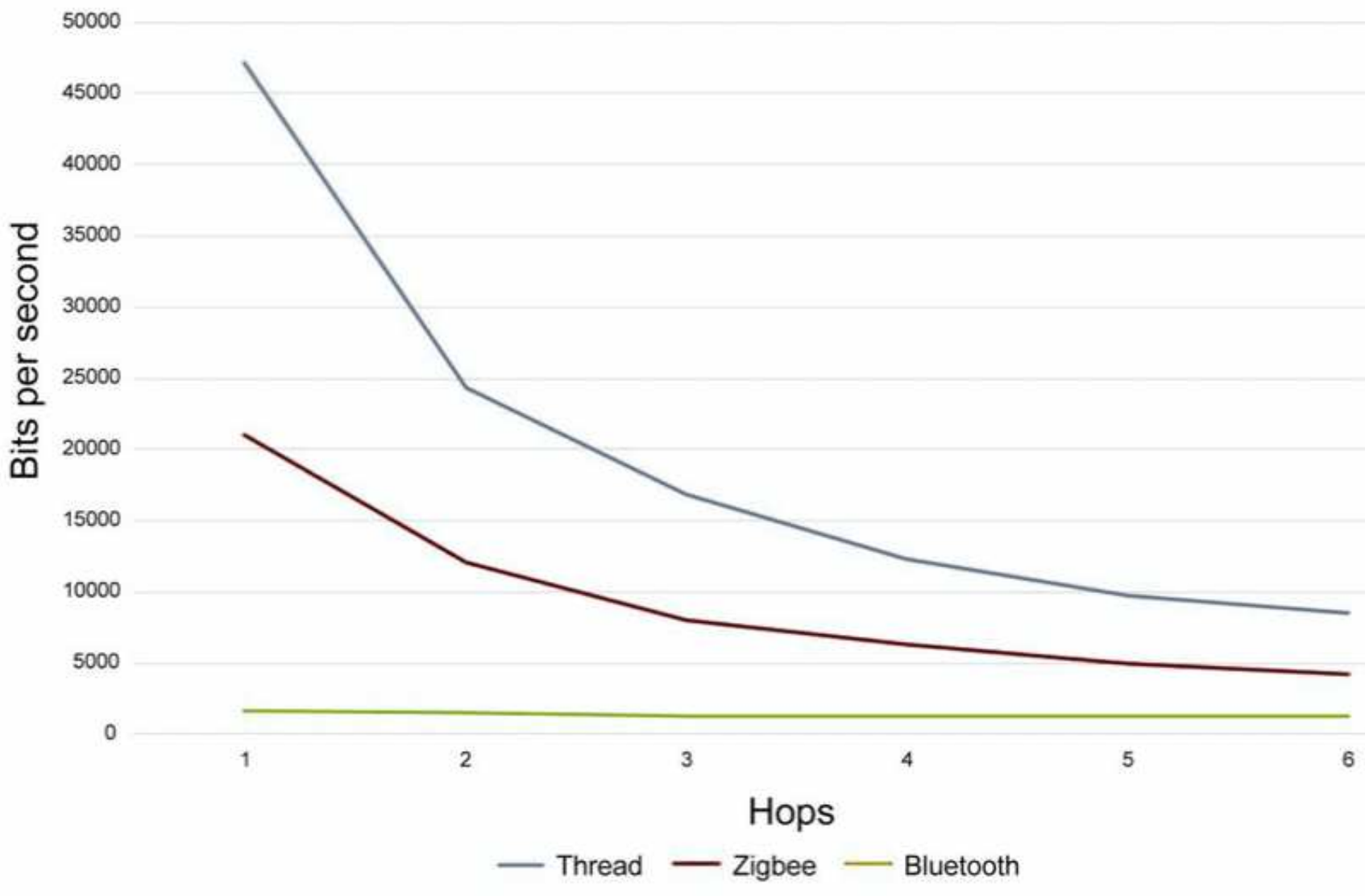

Figure 4 
Cache size comparasion
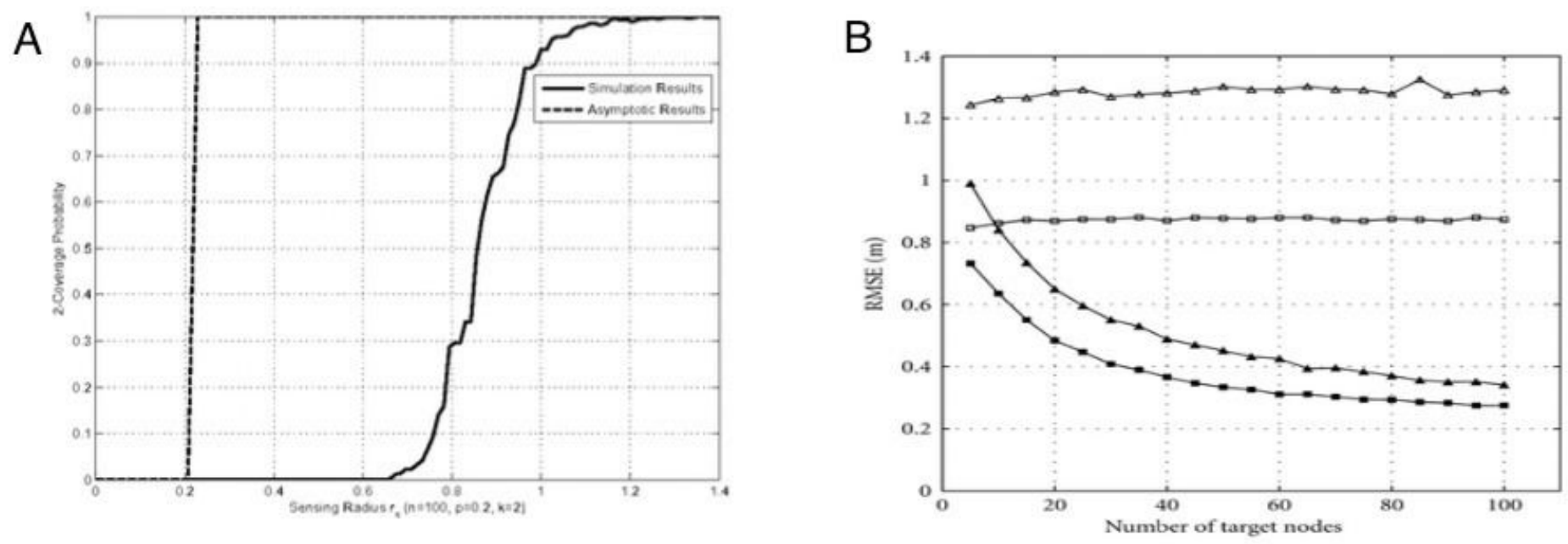

Figure 5

A. Comparison of Energy Consumption in Star/Cluster Network for SDEE routing with and without CCZ caching algorithm. B. Comparison of Energy Consumption in Grid/peer-to-peer Network for SDEE routing with and without CCZ caching algorithm. 